\title{
Generation of excitation signals with prescribed autocorrelation for input and output constrained systems
}

\author{
Christian A. Larsson ${ }^{\dagger}$, Per Hägg ${ }^{\dagger}$ and Håkan Hjalmarsson ${ }^{\dagger}$
}

\begin{abstract}
This paper considers the problem of realizing an input signal with a desired autocorrelation sequence satisfying both input and output constraints for the system it is to be applied to. This is a important problem in system identification. Firstly, the properties of the identified model are highly dependent on the used excitation signal during the experiment and secondly, on real processes, due to actuator saturation and safety considerations, it is important to constrain the inputs and outputs of the process. The proposed method is formulated as a nonlinear model predictive control problem. In general this corresponds to solving a non-convex optimization problem. Here we show how this can be solved in one particular case. For this special case convergence is established for generation of pseudo-white noise. The performance of the algorithm is successfully verified by simulations for a few different autocorrelation sequences, with and without input and output constraints.
\end{abstract}

\section{INTRODUCTION}

O $\mathrm{NE}$ of the most important user choices when making a system identification experiment is the choice of the used input signal. A badly designed input signal could potentially ruin the (in other aspects) most well prepared identification experiment. Conversely, a carefully chosen input signal could reduce the experimental effort needed to get a certain accuracy of the identified model. This understanding has led to the growth of the branch of input or experiment design in system identification [1], [2], [3].

Input design is often formulated as an optimization problem where one tries to minimize an experimental effort, such as input power or experimental length, while fulfilling some specifications on the accuracy of the identified model, or the other way around, given a input power and length of the experiment, find the input signal that minimizes some cost function related to the identified model. These requirements on the model could for example come from the intended use of the model, where a certain accuracy is needed. The outcome of the optimization is often not an input signal but rather an optimal spectrum or an autocorrelation sequence. A problem with optimal input design is that the desired input signal depends on the unknown system, which one wants to identify. One approach to get around this is to make the design robust or insensitive to the true system [4], [5], another is to make the design adaptive, i.e., as information from the system is gathered the input signal properties are changed, see e.g., [6].

The research leading to these results has received funding from the European Union's Seventh Framework Programme (FP7/2007-2013) under grant agreement $\mathrm{n}^{\circ} 257059$, The 'Autoprofit' project (www.fp7-autoprofit.eu) and the Linnaeus Center ACCESS at KTH.

${ }^{\dagger}$ The authors are with the Automatic Control Lab and ACCESS, School of Electrical Engineering, KTH, SE-100 44 Stockholm, Sweden. (e-mails: \{christian.larsson, per.hagg, hjalmars\}@ee.kth.se).
In practical applications there are often constraints on the maximal input and output amplitudes allowed by the process. The input constraint often comes from saturation of the actuator while the output constraint is there to keep the system in a safe operation region. Hence it is important to satisfy these constraints also during the identification experiment.

The question is now: how does one generate a time realization from the given spectrum or autocovariance while satisfying input and output constraints? In the literature, several techniques have been proposed to generate signals with specific properties, see for example [7], [8], [9]. One typical solution is to filter (pseudo random) white noise with unit variance through a spectral factor of the desired spectrum, giving a filtered signal with the desired spectral properties. However with this approach it is difficult to satisfy the input and output amplitude constraints. A second possibility is to consider the class of multi-sines signals [10], [11]. It is straightforward to shape a desired spectrum by a sum of sinusoids with suitable selected amplitudes, since the spectrum of a sine wave signal is a peak at the corresponding frequency. The phase difference between the sine waves can then be designed such that some time domain requirements, for example on the input and output amplitudes, are fulfilled. This often means solving a non-convex optimization problem [12]. A simple heuristic to solve this was proposed in [13] and gives reasonable results in many cases. A third option, which guarantees that the input amplitude constraints are satisfied, is to use a binary input signal. The input signal can then only vary between two different values. There are many proposed methods to realize such a signal, see e.g., [14], [15], [16], [17].

In this paper we propose a novel method of generating signals with prescribed autocorrelation sequence satisfying input and output constraints. The main idea is to, in each time instant, solve a finite horizon optimization problem to find the optimal set of the next, say $N_{u}$, values of the sequence, such that the sampled autocovariance sequence obtained is as close as possible to the desired autocovariance while satisfying input and output signal amplitude constraints for the system the signal is to be applied on. The first term of this optimal solution is then kept and applied to the system. This is repeated until enough samples are generated. This corresponds to a nonlinear Model Predictive Control (MPC) [18] formulation for generating the sequence. This approach is an extension of the work in [16] where binary signals, without output signal amplitude constraints, were considered. The sample by sample calculation of the input signal in the proposed algorithm allows for realtime implementations. This opens up the possibility of utilizing the method in 
adaptive input design. As more and more data are available the identified model of the system gets better. The improved model can then be used to generate a new optimal input autocorrelation using input design.

This paper is outlined as follows. In Section II the algorithm for generation of the input signal is presented. Section III gives a proof of the convergence when the algorithm is used to generate pseudo random white noise. In Section IV the problem with the unknown true system is discussed. The performance is evaluated through simulations in Section V, and finally, Section VI concludes the paper.

\section{Algorithm}

In this section we present the general receding horizon algorithm. The aim is to generate $N$ samples of a signal $U_{N} \triangleq\left[\begin{array}{lll}u_{1} & \ldots & u_{N}\end{array}\right]^{T}$ with prescribed autocorrelation $\mathbf{r}^{d}=\left[\begin{array}{lll}r^{d}(0) & \cdots & r^{d}(n)\end{array}\right]^{T}$ by recursively generating samples while guaranteeing input and output signal amplitude constraints for the system the signal is to be applied on. The (biased) sample autocorrelation of the signal $U_{t}$ is

$$
\begin{aligned}
r_{t}(\tau) & =\frac{1}{t} \sum_{i=\tau+1}^{t} u_{i} u_{i-\tau} \\
& =\frac{t-1}{t} r_{t-1}(\tau)+\frac{1}{t} u_{t} u_{t-\tau} .
\end{aligned}
$$

We choose to minimize the Euclidean distance between the obtained and desired autocovariance sequences, i.e., our goal is to

$$
\underset{U_{N}}{\operatorname{minimize}}\left\|\mathbf{r}_{N}-\mathbf{r}^{d}\right\|_{2}^{2}
$$

where $\mathbf{r}_{t}=\left[\begin{array}{lll}r_{t}(0) & \cdots & r_{t}(n)\end{array}\right]^{T}$ is the sample autocorrelation sequence of the signal $U_{N}$. Now, consider the constrained single input single output (SISO) system

$$
\begin{aligned}
x_{t+1} & =F x_{t}+G u_{t} \\
y_{t} & =H x_{t} \\
u_{\min } \leq u & \leq u_{\max } \\
y_{\min } \leq y & \leq y_{\max } .
\end{aligned}
$$

If the signal, $U_{N}$, generated by (3) is applied to (4) it is desirable that the constraints are satisfied. Notice that the system (4) is deterministic, while in practice there might be disturbances or measurement noise, and the output constraints should hold for the output subject to those disturbances. In other words, the approach presented here only considers constraints on the "noiseless" output. To achieve this we propose the following receding horizon formulation to approximately solve (3). At sample $t$ solve the optimization problem

$$
\begin{array}{cl}
\underset{\tilde{u}_{1}, \ldots \tilde{u}_{N_{u}}}{\operatorname{minimize}} & \left\|\mathbf{r}_{t+N_{u}-1}-\mathbf{r}^{d}\right\|_{2}^{2} \\
\text { subject to } & \hat{x}_{i+1}=F \hat{x}_{i}+G \tilde{u}_{i} \\
& \hat{y}_{i}=H \hat{x}_{i} \\
& \hat{x}_{1}=x_{t} \\
& u_{\min } \leq \tilde{u}_{i} \leq u_{\max }, \quad i=1 \ldots N_{u} \\
& y_{\min } \leq \hat{y}_{i} \leq y_{\max }, \quad i=1 \ldots N_{y} .
\end{array}
$$

Only the first element of the optimal solution is used, i.e., $u_{t}=\tilde{u}_{1}^{\star}$, where $\star$ denotes the optimal value. The optimization is performed iteratively in receding horizon fashion. We will refer to $N_{u}$ and $N_{y}$ as the input and output horizons, respectively. The input horizon is the number of samples in $U_{N}$ that are optimized in each time step while the output horizon is how far in the future we consider that the output should satisfy the constraints. If $N_{u}<N_{y}$ set $u_{N_{u}+1}=\cdots=u_{N_{y}}=0$, i.e., if the output horizon is longer than the input horizon the input is considered zero over the remaining part of the horizon. We introduce the notation $\mathbf{0}_{n}$ for the zero vector with $n$ elements.

The steps of the complete algorithm are:

1) Set $t=1, x_{0}=x$ and $\mathbf{r}_{0}=\mathbf{0}_{n+1}$.

2) Update $\mathbf{r}_{t}$ according to (2).

3) Solve the optimization problem (5). If the solution is non unique, arbitrarily choose the optimum $u^{\star}$.

4) Set $u_{t}=\tilde{u}_{1}^{\star}$.

5) Set $x_{t+1}=F x_{t}+G u_{1}^{\star}$.

6) If $t<N, t \leftarrow t+1$ and go to Step 2; otherwise stop.

This formulation of the signal generation can be seen as a nonlinear MPC for the system described by the recursion of the sample autocovariance (2). The vector $\mathbf{r}_{t}$ can be seen as the state vector and the goal is to drive the states to the desired autocorrelation sequence while satisfying the system constraints (4).

A problem that can occur with the constrained optimization problem (5) is that it may be infeasible. But one way or another the signal has to be generated at each time step. This is a well known problem in Model Predictive Control and various approaches to overcome this has been suggested. One could for example avoid the hard constraints or change the constraint at each time instant to get a feasible problem, see [18] for details. We will use a simpler solution, if it is not possible to satisfy a constraint we just ignore it. If this is a good approach depends on what one prioritizes.

In general, the optimization problem (5) is non-convex and difficult to solve. There are commercially available tools and software for nonlinear MPC, for an overview of existing tools see for example [19], [20]. However, for the choice of $N_{u}=1$ the optimization can be done analytically. This is shown in the following sub-section.

\section{A. Input horizon $N_{u}=1$}

For the specific case of $N_{u}=1$, the solution to (5) is particularly simple. First notice that the objective function at sample $t$ can be written as

$$
\begin{aligned}
\left\|\mathbf{r}_{t}-\mathbf{r}^{d}\right\|_{2}^{2}= & \\
& \begin{array}{r}
\left.\frac{t-1}{t} \mathbf{r}_{t-1}+\frac{1}{t} u_{t}\left[\begin{array}{c}
u_{t} \\
U_{t-1}
\end{array}\right]-\mathbf{r}^{d}\right)^{T} \times \\
\left(\frac{t-1}{t} \mathbf{r}_{t-1}+\frac{1}{t} u_{t}\left[\begin{array}{c}
u_{t} \\
U_{t-1}
\end{array}\right]-\mathbf{r}^{d}\right) .
\end{array}
\end{aligned}
$$

This is a polynomial of degree 4 in the single optimization variable $u_{t}$ and all other quantities are fixed. Hence the optimal value can be found analytically by differentiating (6) with respect to $u_{t}$. The optimum is attained either at the zeros of the derivative or at one of the constraints on 
$u$. Since the derivative is a $3^{\text {rd }}$ order polynomial there exist closed form expressions for the zeros [21].

\section{CONVERGENCE}

In this section the convergence of the algorithm is proved for the special case of generating pseudo random white noise, with input horizon $N_{u}=1$ and no output constraints from the system, hence we set the output horizon to $N_{y}=0$. The convergence of the algorithm is established in the following theorem.

Theorem 1: For the algorithm (5) with

$$
\mathbf{r}^{d}=\left[\begin{array}{llll}
\sigma^{2} & 0 & \ldots & 0
\end{array}\right]^{T}
$$

i.e., generation of pseudo white noise with variance $\sigma^{2}$, and

$$
u_{\max } \geq \sigma, \quad u_{\min }=-u_{\max }
$$

it holds that

$$
\lim _{t \rightarrow \infty} \frac{1}{t} \sum_{i=\tau}^{t} u_{i} u_{i-\tau}=r^{d}(\tau), \quad \tau=0, \ldots, n .
$$

Proof: The proof is very similar to the convergence proof provided in [16].

Introduce

$$
\tilde{\mathbf{r}}_{t}=\left[\begin{array}{lll}
\tilde{r}_{t}(0) & \cdots & \tilde{r}_{t}(n)
\end{array}\right]^{T}
$$

where

$$
\tilde{r}_{t}(\tau)=\sum_{i=\tau+1}^{t}\left(u_{i} u_{i-\tau}-r^{d}(\tau)\right), \quad 0 \leq \tau \leq \min (t-1, n) .
$$

Then

$$
\tilde{r}_{t+1}(\tau)=\tilde{r}_{t}(\tau)+u_{t} u_{t-\tau}-r^{d}(\tau)
$$

allowing the dynamics of $\tilde{\mathbf{r}}_{t}$ to be written as

$$
\tilde{\mathbf{r}}_{t+1}=\tilde{\mathbf{r}}_{t}+u_{t} \mathbf{u}_{t}-\tilde{\mathbf{r}}^{d}
$$

where $\mathbf{u}_{t}=\left[\begin{array}{llll}u_{t} & u_{t-1} & \ldots & u_{t-n}\end{array}\right]^{T}$, with initial condition

$$
\tilde{\mathbf{r}}_{0}=\mathbf{0}_{n+1} \text {. }
$$

The goal is hence to show that

We note that

$$
\lim _{t \rightarrow \infty} \frac{\tilde{\mathbf{r}}_{t}}{t}=\mathbf{0}_{n+1} \text {. }
$$

$$
u_{t}=\arg \min _{u_{t}}\left\|\tilde{\mathbf{r}}_{t+1}\right\|_{2}^{2}
$$

Hence

$$
\begin{aligned}
& \left\|\tilde{\mathbf{r}}_{t+1}\right\|_{2}^{2}=\min _{u_{t}}\left\|\tilde{\mathbf{r}}_{t+1}\right\|_{2}^{2} \\
& =\min _{u_{t}}\left\{\left(\tilde{r}_{t}(0)+u_{t}^{2}-\sigma^{2}\right)^{2}+\sum_{\tau=1}^{n}\left(\tilde{r}_{t}(\tau)+u_{t} u_{t-\tau}\right)^{2}\right\} \\
& \leq \min _{u_{t} \in\{-\sigma, \sigma\}}\left\{\left(\tilde{r}_{t}(0)+u_{t}^{2}-\sigma^{2}\right)^{2}+\sum_{\tau=1}^{n}\left(\tilde{r}_{t}(\tau)+u_{t} u_{t-\tau}\right)^{2}\right\} \\
& =\tilde{r}_{t}(0)^{2}+\min _{u_{t} \in\{-\sigma, \sigma\}} \sum_{\tau=1}^{n}\left(\tilde{r}_{t}(\tau)+u_{t} u_{t-\tau}\right)^{2} \\
& \leq\left\|\tilde{\mathbf{r}}_{t}\right\|_{2}^{2}+\sum_{\tau=1}^{n} \sigma^{2} u_{\max }^{2} \\
& \leq\left\|\tilde{\mathbf{r}}_{t}\right\|_{2}^{2}+n \sigma^{2} u_{\max }^{2}, \quad t>n
\end{aligned}
$$

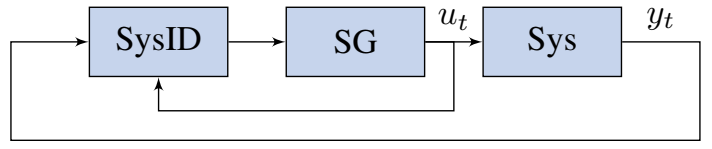

Fig. 1: Adaptive signal generation scheme.

Iterating (7) over $t \in \mathbb{N}$ using that $\tilde{\mathbf{r}}_{0}=\mathbf{0}_{n+1}$ gives

$$
\left\|\tilde{\mathbf{r}}_{t}\right\|_{2}^{2} \leq n u_{\max }^{4} t+c, \quad t>n
$$

where $c$ is an upper bound on $\sum_{t=1}^{n}\left\|\tilde{\mathbf{r}}_{t}\right\|_{2}^{2}$. Noting that

$$
\begin{aligned}
\left\|\tilde{\mathbf{r}}_{t}\right\|_{2}^{2} & =\left(\sum_{i=1}^{t}\left(u_{i}^{2}-\sigma^{2}\right)\right)^{2}+\sum_{\tau=1}^{n}\left(\sum_{i=\tau+1}^{t}\left(u_{i} u_{i-\tau}\right)\right)^{2} \\
& \leq\left(\sum_{i=\tau+1}^{t} u_{i}^{2}\right)^{2}+\left(\sum_{i=\tau+1}^{t} \sigma^{2}\right)^{2}+\sum_{\tau=1}^{n}\left(t u_{\max }^{2}\right)^{2} \\
& \leq t^{2} u_{\max }^{4}+n t^{2} u_{\max }^{4}=(n+1) t^{2} u_{\max }^{4} .
\end{aligned}
$$

the bound, $c$, can be derived from

$\sum_{t=1}^{n}\left\|\tilde{\mathbf{r}}_{t}\right\|_{2}^{2} \leq(n+1) u_{\max }^{4} \sum_{t=1}^{n} t^{2}=\frac{n(n+1)^{2}(2 n+1)}{6} u_{\max }^{4}$.

Dividing (8) by $t^{2}$ gives for $t=n+1, \ldots$

$$
\sum_{\tau=0}^{n}\left[\frac{1}{t} \sum_{i=\tau+1}^{t}\left(u_{i} u_{i-\tau}-r^{d}(\tau)\right)\right]^{2} \leq \frac{n u_{\max }^{4}}{t}+\frac{c}{t^{2}},
$$

and consequently

$$
\left|\frac{1}{t} \sum_{i=\tau+1}^{t}\left(u_{i} u_{i-\tau}-r^{d}(\tau)\right)\right| \leq \sqrt{\frac{n u_{\max }^{4}}{t}+\frac{c}{t^{2}}} .
$$

Since

$$
\lim _{t \rightarrow \infty} \sqrt{\frac{n u_{\max }^{4}}{t}+\frac{c}{t^{2}}}=0
$$

we conclude that

$$
\lim _{t \rightarrow \infty} \frac{1}{t} \sum_{i=\tau+1}^{t} u_{i} u_{i-\tau}=r^{d}(\tau)
$$

\section{The UnKNOWN TRUE SYSTEM}

The proposed method in this paper requires perfect knowledge of the true system to be able to predict the output. However, this contradicts the purpose of the method: to generate input signals that can be used to identify the system. Here we outline two ways to get around this fundamental problem.

The first approach is to make the method adaptive. An example of how this can be achieved is shown in Figure 1. The output $y_{t}$ from the true system (Sys) is measured and used together with the input $u_{t}$ to estimate a model and the current state of the system in the system identification block (SysID). The estimated model and state of the system are then used in the signal generation (SG) algorithm presented earlier. This is repeated and as the estimated model is 


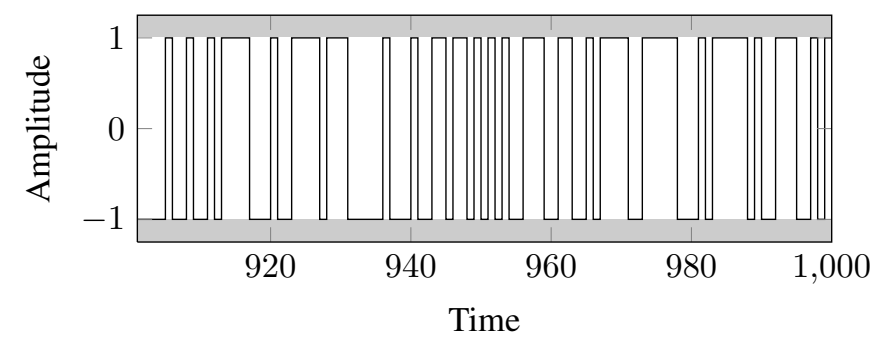

(a) Generated signal, $u(t)$ for $t=901, \ldots, 1000$.

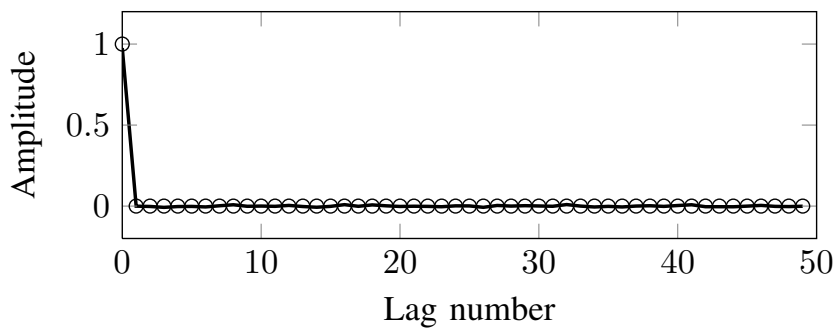

(b) Desired autocovariance (O) and autocovariance of the generated signal (一).

Fig. 2: Generation of a pseudo random white noise sequence with amplitude constraints, \pm 1 .

improved the predicted output from the system gets more accurate.

The second approach is to use robust signal generation. Assume that an initial model is known within some uncertainty region. We could then generate the input samples while satisfy the output constraints, not just based on the initial model, but for all possible models in the uncertainty region. This is related to robust MPC, see [18]. This approach could give quite conservative bounds on $u_{t}$ if the initial model is uncertain.

\section{EXAMPLES}

In this section we present three examples. The first example shows generation of a pseudo random white noise sequence. The proposed algorithm is also compared to the algorithm suggested in [16]. The second example deals with signal generation under amplitude constraints which is an important issue in, e.g. system identification. The third example shows that the algorithm can be applied to autocorrelation sequences corresponding to colored noise.

\section{A. Pseudo random white noise}

Here the aim is to generate $U_{1}^{N}$ with a desired autocorrelation sequence $\mathbf{r}^{d}$ where the first element is the only non-zero entry. We will match $n=50$ autocorrelation coefficients. The input horizon is set to $N_{u}=1$ and the amplitude of the signal is constrained to be inside \pm 1 . Since we do not consider any output constraints in this example, we set the output horizon to $N_{y}=0$. The number of generated samples is $N=10^{3}$. In Figure 2 the autocorrelation and the power spectral density of the generated signal are shown. The characteristics of the signal are very close to those of white noise.

The convergence of the algorithm is illustrated in Figure 3, where the dependence of the cost function on the number of samples $N$ is shown. A total of $N=10^{6}$ samples

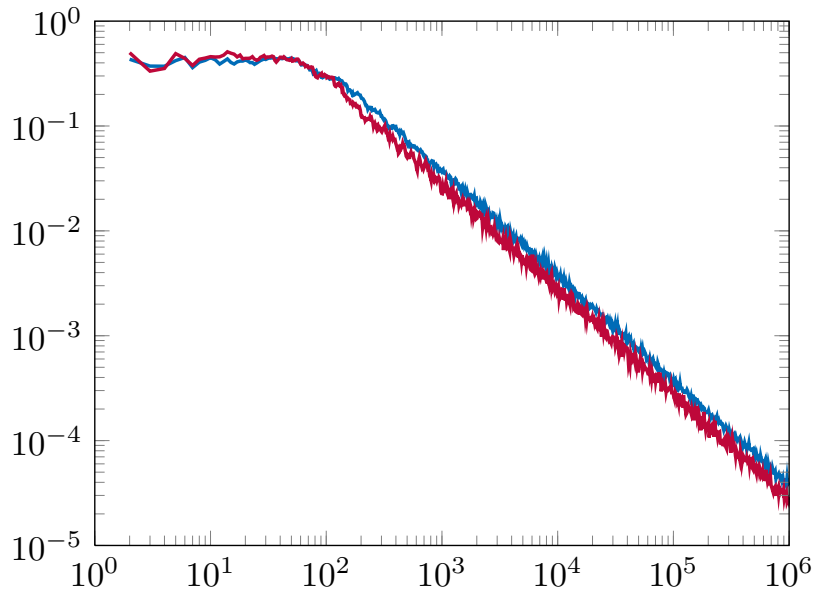

Fig. 3: Convergence rate for the proposed method (-) and the method from [16] (-) for generation of pseudo random white noise.

is generated and after each sample the autocovariance is calculated and compared to the desired one. The measure of the error at time $t$ is

$$
\epsilon(t)=\sqrt{\sum_{\tau=0}^{n}\left(r_{u}^{t}(\tau)-r^{d}\right)^{2}}
$$

where $r_{u}^{t}(\tau)$ is the autocovariance sequence calculated with data up to time $t$. The convergence rates seems to be $\mathcal{O}(1 / N)$. For comparison, the cost function of the algorithm in [16] is also shown. The convergence rates of the two methods are close to each other. This is expected since the signal of the proposed method becomes a binary signal in this case. However, the algorithm in [16] requires a much smaller run time to generate the same number of samples.

\section{B. System with constraints}

In this example we consider generation of a signal where we want the output of a system, on which the signal is applied, to be constrained. The considered system is of order 20 and generated using the drss command in Matlab. The direct term from input to output is removed and the system is normalized to have 2-norm equal to 1 . We would like to generate a white noise sequence with maximum amplitude of 2 while keeping the output of the system inside \pm 1.5 . The settings used are $N=1000, N_{u}=1$, and $n=50$. Three different output horizons are considered: $N_{y}=0$ (i.e., the output constraints are not considered), $N_{y}=1$ and $N_{y}=5$. The resulting outputs are shown in Figure 4. For the first case when $N_{y}=0$ the constraint is violated in 130 of the 1000 samples, for $N_{y}=1$ it is violated at 70 samples and for the longest horizon, $N_{y}=5$ there are no violations of the constraints. Hence the longer output horizon the more conservative the algorithm is when choosing $u$, making it possible to satisfy the constraints. However, choosing the horizon too long might (for certain systems, such as unstable systems or systems close to being unstable) render an autocorrelation of the generated sequence far from the desired one. 


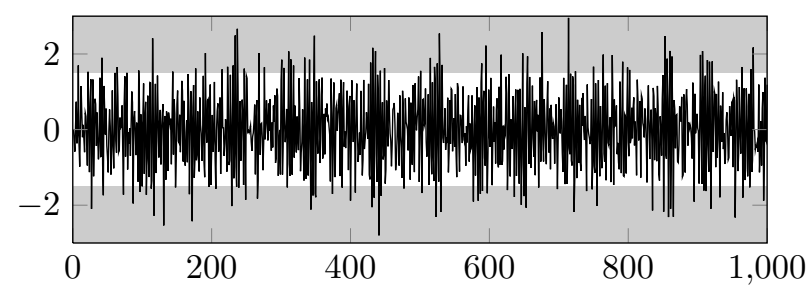

(a) $N_{y}=0$.

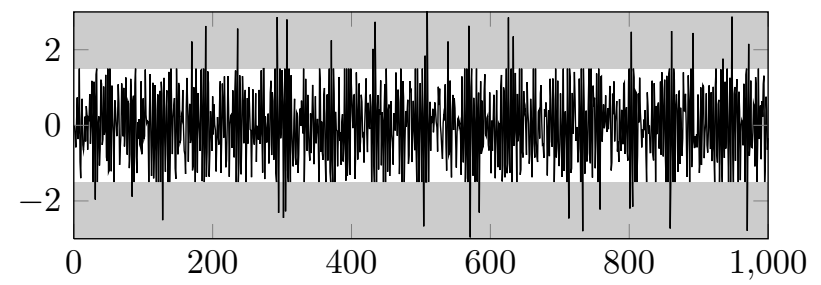

(b) $N_{y}=1$.

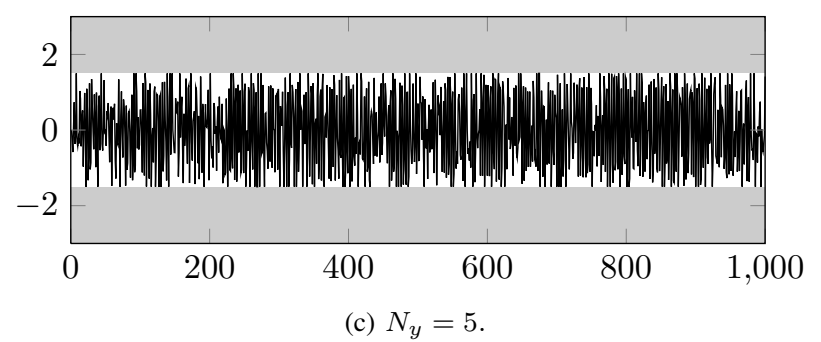

Fig. 4: Output from the system for the three different generated signals.

The convergence results for the three cases are shown in Figure 5. It is seen that the cost of longer output horizon, i.e., to satisfy the output constraints better, is slower convergence. However in this case the cost is quite low.

\section{AR(1)-process}

This example illustrates the applicability of the algorithm to generate signals with non white autocorrelations with both input and output constraints. The system that the generated signal is to be applied to is the FIR system

$$
G(z)=0.3 z^{-1}+0.425 z^{-2}+0.2125 z^{-3}+0.1875 z^{-4}
$$

with a non minimum phase zero at $z_{n m p}=-1.25$. Assume that the goal is to identify the location of this zero, then the input signal that minimizes the variance of the estimate is given by the $\mathrm{AR}(1)$ process, see [22],

$$
x_{t+1}=a x_{t}+e_{t},
$$

where $e_{t}$ is a white noise signal and $a=z_{n m p}^{-1}=-0.8$. The autocorrelation is given by

$$
E\left\{x_{t} x_{t-\tau}\right\}=\frac{a^{|\tau|}}{1-a^{2}} .
$$

We set $N=1000, N_{u}=1$, and $n=50$. The generated signal is constrained to have amplitude inside \pm 1.5 and the output should be inside \pm 0.5 . Three different length of the output horizon are considered, $N_{y}=0,1,2$. The resulting output signals from the system are shown in Figure 6. For

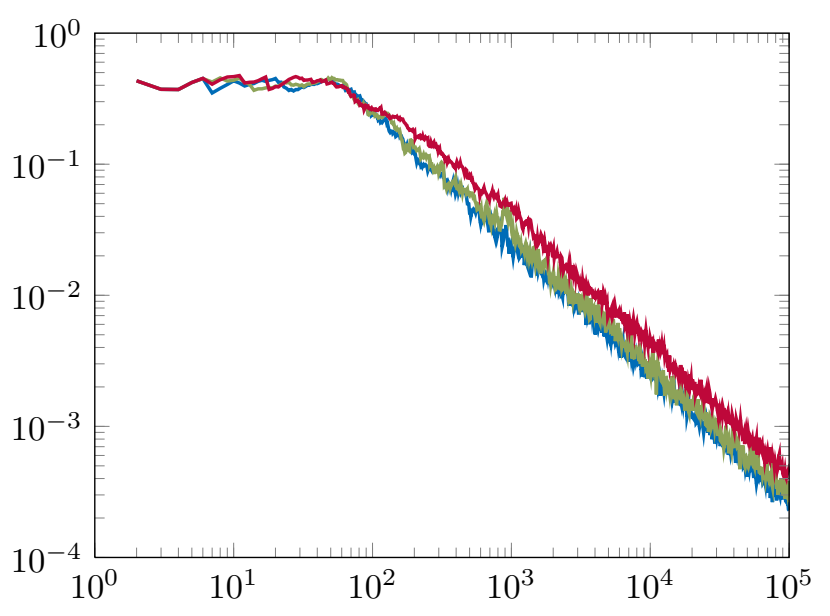

Fig. 5: Convergence rate for generation of pseudo-white noise for three different output horizons $N_{y}=0$ (-), $N_{y}=1(-)$ and $N_{y}=5$ ( $(-)$.

$N_{y}=0$ the output signal violates the output constraint 57 times while the constraint never is violated for the longer output horizons. However, for the case $N_{y}=2$ the horizon is too long and the solution is conservative, the generated signals amplitude becomes less than 1.1 for all time. This leads to an autocorrelation far from the desired one.

Again the convergence rate is studied. The results are illustrated in Figure 7. The convergence for the case $N_{y}=0$ and $N_{y}=1$ are similar and the cost of imposing the output constraint is low. For $N_{y}=2$ the solution is too conservative to satisfy the desired autocorrelation and the method does not converge in this case.

\section{CONCLUSIONS}

In this paper we presented a novel method for generation of input signals with a specified sample autocovariance while satisfying input and output constraints of the system the signal are to be applied on. The method corresponds to a nonlinear MPC problem, which in general leads to a non-convex optimization problem. However, it was shown that an analytic solution is available in one particular case. For this case convergence was established for generation of pseudo-white noise. Furthermore, simulations were used to verify the performance of the algorithm for a few different autocorrelation sequences, with and without input and output constraints.

The method relies on perfect knowledge of the true system. Two approaches to get around this problem were outlined and should be further investigated.

The formulation of the method allows for realtime implementations. It would be interesting to see if this algorithm can be used in closed loop, simultaneously estimating the model used to control the system to satisfy the constraint and updating the desired input signal properties using input design.

A more general question is what kind of autocovariance sequences the proposed method can realize. This is related to the theoretical problem of finding the minimum crest factor, i.e., the minimum amplitude for which the desired 


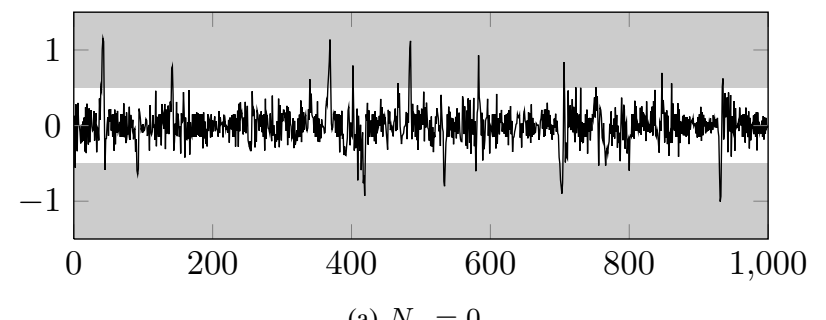

(a) $N_{y}=0$.

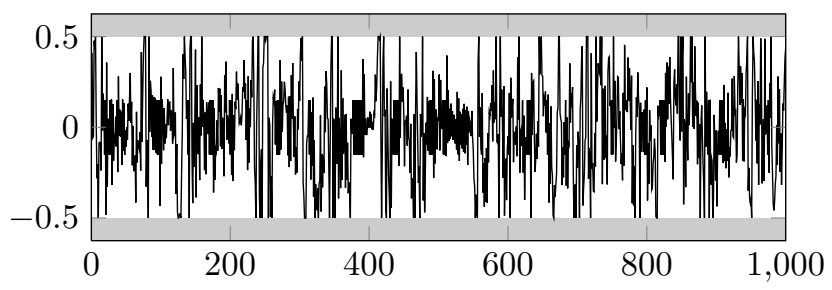

(b) $N_{y}=1$.

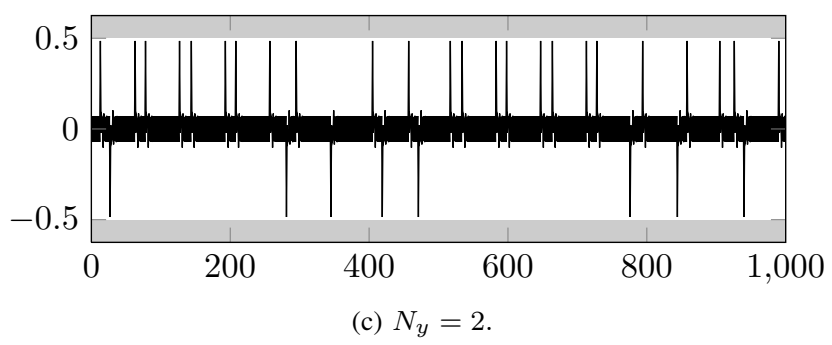

Fig. 6: Output from the FIR-system for the three different generated signals.

autocovariance can be realized, an interesting problem for future work.

\section{REFERENCES}

[1] V. Fedorov, Theory of optimal experiments, ser. Probability and mathematical statistics. Academic Press, 1972.

[2] G. Goodwin and R. Payne, Dynamic system identification: experiment design and data analysis, ser. Mathematics in science and engineering. Academic Press, 1977.

[3] L. Ljung, System Identification: Theory for the User, 2nd Edition. Upper Saddle River, New Jersey: Prentice Hall, 1999.

[4] C. R. Rojas, J. S. Welsh, G. C. Goodwin, and A. Feuer, "Robust optimal experiment design for system identification," Automatica, vol. 43, no. 6, pp. $993-1008,2007$.

[5] C. A. Larsson, E. Geerardyn, and J. Schoukens, "Robust optimal input design," in IFAC Symposium on System Identification (SYSID 2012, Brussels), 2102.

[6] L. Gerencsér and H. Hjalmarsson, "Adaptive input design in system identification," in Decision and Control, 2005 and 2005 European Control Conference. CDC-ECC '05. 44th IEEE Conference on, dec. 2005, pp. $4988-4993$.

[7] K. Godfrey, Perturbation signals for system identification, ser. Prentice Hall international series in acoustics, speech, and signal processing. Prentice Hall, 1993.

[8] U. Gujar and R. Kavanagh, "Generation of random signals with specified probability density functions and power density spectra," Automatic Control, IEEE Transactions on, vol. 13, no. 6, pp. 716 - 719, dec 1968.

[9] B. Liu and J. Munson, D., "Generation of a random sequence having a jointly specified marginal distribution and autocovariance," Acoustics, Speech and Signal Processing, IEEE Transactions on, vol. 30, no. 6, pp. $973-983$, dec 1982.

[10] R. Pintelon and J. Schoukens, System identification : a frequency domain approach. New York: IEEE Press, 2001.

[11] J. Schoukens, P. Guillaume, and R. Pintelon, "Design of multisine excitations," in Control 1991. Control '91., International Conference on, mar 1991, pp. 638 -643 vol.1.

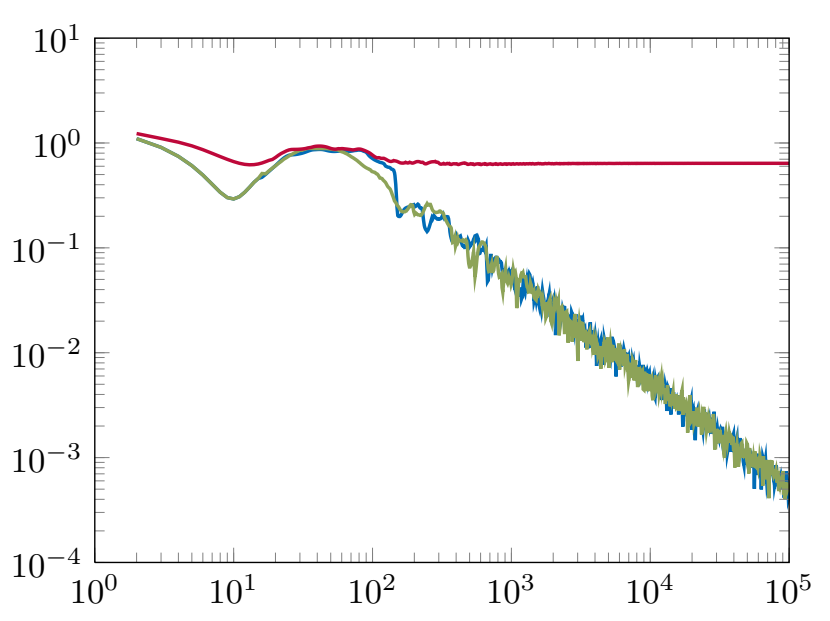

Fig. 7: Convergence rate for generation of an AR(1)-process with input and output constraints for three different output horizons $N_{y}=0(-), N_{y}=1$ ( $(-)$ and $N_{y}=2(\square)$.

[12] D. E. Rivera, H. Lee, H. D. Mittelmann, and M. W. Braun, "Constrained multisine input signals for plant-friendly identification of chemical process systems," Journal of Process Control, vol. 19, no. 4, pp. $623-635,2009$.

[13] M. Schroeder, "Synthesis of low-peak-factor signals and binary sequences with low autocorrelation (corresp.)," Information Theory, IEEE Transactions on, vol. 16, no. 1, pp. 85 - 89, jan 1970.

[14] A. H. Tan and K. Godfrey, "The generation of binary and near-binary pseudorandom signals: an overview," Instrumentation and Measurement, IEEE Transactions on, vol. 51, no. 4, pp. 583 - 588, aug 2002.

[15] P.-S. Koutsourelakis and G. Deodatis, "Simulation of binary random fields with applications to two-phase random media," Journal of Engineering Mechanics, vol. 131, no. 4, pp. 397-412, 2005.

[16] C. R. Rojas, J. Welsh, and G. Goodwin, "A receding horizon algorithm to generate binary signals with a prescribed autocovariance," in American Control Conference, 2007. ACC '07, july 2007, pp. 122 -127 .

[17] C. Brighenti, B. Wahlberg, and C. R. Rojas, "Input design using markov chains for system identification," in Decision and Control, 2009 held jointly with the 2009 28th Chinese Control Conference. CDC/CCC 2009. Proceedings of the 48th IEEE Conference on, dec. 2009, pp. $1557-1562$.

[18] J. Maciejowski, Predictive control: with constraints, ser. Pearson Education. Prentice Hall, 2002.

[19] S. Qin and T. A. Badgwell, "A survey of industrial model predictive control technology," Control Engineering Practice, vol. 11, no. 7, pp. $733-764,2003$.

[20] S. J. Qin and T. A. Badgwell, "An overview of nonlinear model predictive control applications," in Nonlinear Predictive Control. Verlag, 2000, pp. 369-392.

[21] L. Guilbeau, "The history of the solution of the cubic equation," Mathematics News Letter, vol. 5, no. 4, pp. 8-12, 1930.

[22] J. Mårtensson, H. Jansson, and H. Hjalmarsson, "Input design for identification of zeros," in Proceedings of the 16th IFAC World Congress, 2005, 2005. 